\title{
Intra-Aortic Balloon Pump
}

National Cancer Institute

\section{Source}

National Cancer Institute. Intra-Aortic Balloon Pump. NCI Thesaurus. Code C100087.

A medical device that increases myocardial oxygen perfusion while at the same time increasing cardiac output. (ACC) 